\title{
Integrin AlphaV/Beta6
}

National Cancer Institute

\section{Source}

National Cancer Institute. Integrin AlphaV/Beta6. NCI Thesaurus. Code C124258.

Integ rin alphaV/beta6 is a protein complex comprised of a heterodimer of integ rin alpha-

$\checkmark$ and integrin beta-6. This complex plays a role in cell-matrix adhesion and viral entry. 